\title{
A class of small deviation theorems for the random fields on an $m$ rooted Cayley tree
}

\author{
Zhiyan Shi ${ }^{1 *}$, Weiguo Yang ${ }^{1}$, Lixin $\operatorname{Tian}^{1}$ and Weicai Peng ${ }^{2}$
}

\author{
* Correspondence: \\ shizhiyan1984@126.com \\ ${ }^{1}$ Faculty of Science, Jiangsu \\ University, Zhenjiang 212013, China \\ Full list of author information is \\ available at the end of the article
}

\begin{abstract}
In this paper, we are to establish a class of strong deviation theorems for the random fields relative to $m$ th-order nonhomogeneous Markov chains indexed by an $m$ rooted Cayley tree. As corollaries, we obtain the strong law of large numbers and Shannon-McMillan theorem for mth-order nonhomogeneous Markov chains indexed by that tree.
\end{abstract}

2000 Mathematics Subject Classification: 60F15; 60J10.

Keywords: strong deviation theorem, $m$ rooted Cayley tree, $m$ th-order nonhomogeneous Markov chain, Shannon-McMillan theorem

\section{Introduction}

A tree is a graph $G=\{T, E\}$ which is connected and contains no circuits. Given any two vertices $\sigma, t(\sigma \neq t \in T)$, let $\overline{\sigma t}$ be the unique path connecting $\sigma$ and $t$. Define the graph distance $d(\sigma, t)$ to be the number of edges contained in the path $\overline{\sigma t}$.

Let $T_{C, N}$ be a Cayley tree. In this tree, the root (denoted by $o$ ) has only $N$ neighbors and all other vertices have $N+1$ neighbors. Let $T_{B, N}$ be a Bethe tree, on which each vertex has $N+1$ neighboring vertices. Here both $T_{C, N}$ and $T_{B, N}$ are homogeneous tree. In this paper, we mainly consider an $m$ rooted Cayley tree $\bar{T}_{C, N}$ (see Figure 1 ). It is formed by a Cayley tree $T_{C, N}$ with the root $o$ connecting with another vertex denoted by the the root -1 , and then root -1 connecting with another vertex denoted by the root -2 , and continuing to do the same work until the last vertex denoted by the root - $(m-1)$ is connected. When the context permits, this type of tree is denoted simply by $T$.

Let $\sigma, t(\sigma, t \neq o,-1,-2, \ldots,-(m-1))$ be vertices of an $m$ rooted Cayler tree $T$. Write $t$ $\leq \sigma$ if $t$ is on the unique path connecting $o$ to $\sigma$, and $|\sigma|$ the number of edges on this path. For any two vertices $\sigma, t(\sigma, t \neq o,-1,-2, \ldots,-(m-1))$ of tree $T$, denote by $\sigma \wedge t$ the vertex farthest from $o$ satisfying $\sigma \wedge t \leq \sigma$ and $\sigma \wedge t \leq t$.

The set of all vertices with distance $n$ from the root $o$ is called the $n$-th generation of $T$, which is denoted by $L_{n}$. We say that $L_{n}$ is the set of all vertices on level $n$ and especially root -1 is on the -1 st level on tree $T$, root -2 is on the -2 nd level. By analogy, root $-(m-1)$ is on the $-(m-1)$ th level. We denote by $T^{(n)}$ the subtree of an $m$ rooted Cayley tree $T$ containing the vertices from level $-(m-1)$ (the root $-(m-1))$ to level $n$. Let $t(t \neq o,-1,-2, \ldots,-(m-1))$ be a vertex of an $m$ rooted Cayley tree $T$. Predecessor of the vertex $t$ is another vertex, which is nearest from $t$, on the unique path from root $-(m-1)$ to $t$. We denote the predecessor of $\mathrm{t}$ by $1_{t}$, the predecessor of $1_{t}$ by $2_{t}$ and the

(C) 2012 Shi et al; licensee Springer. This is an Open Access article distributed under the terms of the Creative Commons Attribution License (http://creativecommons.org/licenses/by/2.0), which permits unrestricted use, distribution, and reproduction in any medium, provided the original work is properly cited. 


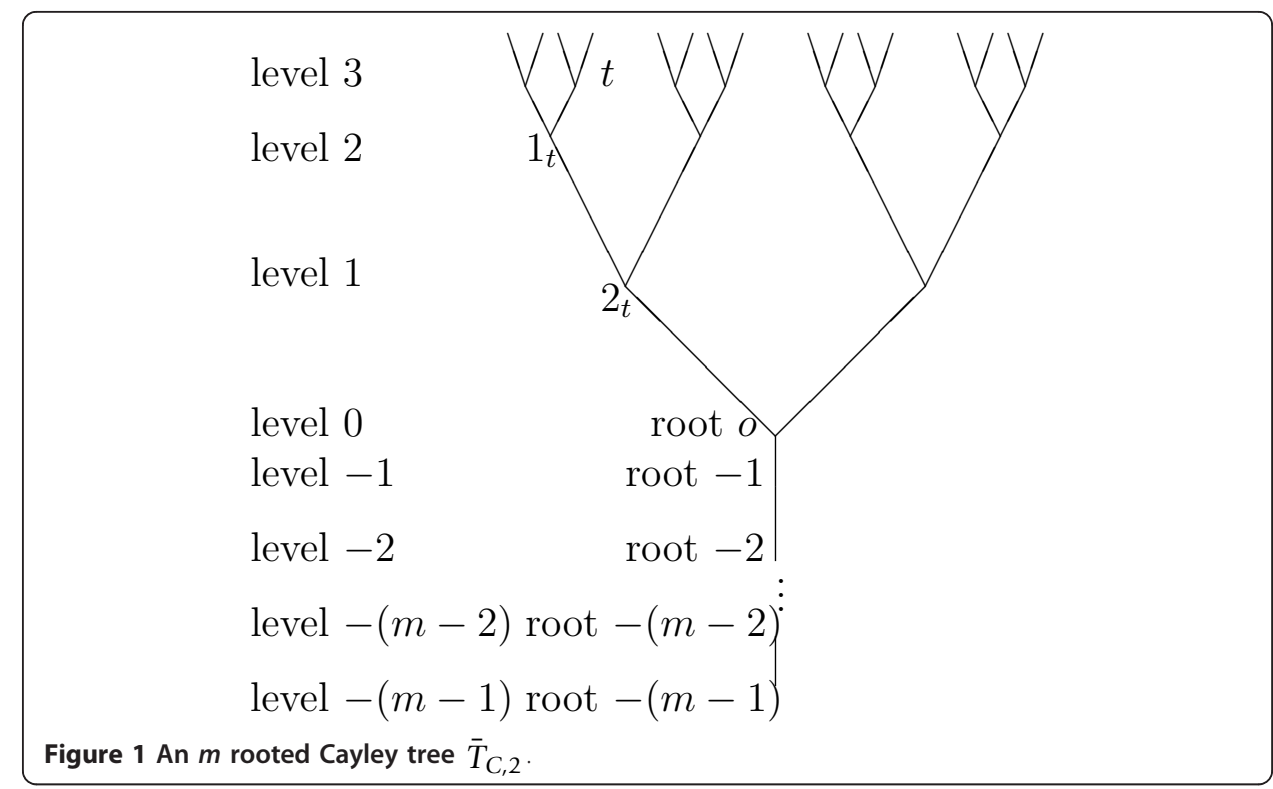

predecessor of $(n-1)_{t}$ by $n_{t}$. We also say that $n_{t}$ is the $n$-th predecessor of $t . X^{A}=\left\{X_{t}\right.$, $t \in A\}$ is a stochastic process indexed by a set $A$, and denoted by $|A|$ the number of vertices of $A, x^{A}$ is the realization of $X^{A}$.

Let $(\Omega, \mathcal{F})$ be a measure space, $\left\{X_{t}, t \in T\right\}$ be a collection of random variables defined on $(\Omega, \mathcal{F})$ and taking values in $G=\{0,1, \ldots, b-1\}$, where $b$ is a positive integer. Let $P$ be a general probability distribution on $(\Omega, \mathcal{F})$. We will call $P$ the random field on tree $T$. Denote the distribution of $\left\{X_{t}, t \in T\right\}$ under the probability measure $P$ by

$$
P\left(x^{T^{(n)}}\right)=P\left(X^{T^{(n)}}=x^{T^{(n)}}\right), \quad x^{T^{(n)}} \in G^{T^{(n)}} .
$$

Let

$$
f_{n}(\omega)=-\frac{1}{\left|T^{(n)}\right|} \ln P\left(X^{T^{(n)}}\right) .
$$

$f_{n}(\omega)$ is called entropy density of $X^{T^{(n)}}$.

Let $Q$ be another probability measure on the measurable space $(\Omega, \mathcal{F})$, and let the distribution of $\left\{X_{t}, t \in T\right\}$ under $Q$ be

$$
Q\left(x^{T^{(n)}}\right)=Q\left(X^{T^{(n)}}=x^{T^{(n)}}\right), \quad x^{T^{(n)}} \in G^{T^{(n)}} .
$$

Let

$$
h(P \mid Q)=\limsup _{n \rightarrow \infty} \frac{1}{\left|T^{(n)}\right|} \ln \frac{P\left(X^{T^{(n)}}\right)}{Q\left(X^{T^{(n)}}\right)} .
$$

$h(P \mid Q)$ is called the sample divergence rate of $P$ relative to $Q$.

Remark 1 If $P=Q, h(P \mid Q)=0$ holds. By using the approach of Lemma 1 of Liu and Wang [1], we also can prove that $h(P \mid Q) \geq 0, P$ - a.e.; hence, $h(P \mid Q)$ can be regarded as a measure of the Markov approximation of the arbitrary random field on $T$.

Definition 1 (see [2]) Let $G=\{0,1, \ldots, b-1\}$ and $P\left(y \mid x_{1}, x_{2}, \ldots, x_{m}\right)$ be a nonnegative functions on $G^{m+1}$. Let 
If

$$
\sum_{y \in G} P\left(y \mid x_{1}, x_{2}, \ldots, x_{m}\right)=1
$$

then $P$ is called an $m$-order transition matrix.

Definition 2 (see [2]). Let $T$ be an $m$ rooted Cayley tree, and let $G=\{0,1, \ldots, b-1\}$ be a finite state space, $\left\{X_{t}, t \in T\right\}$ be a collection of $G$-valued random variables defined on the probability space $(\Omega, \mathcal{F}, Q)$. Let $Q$ be a probability on a measurable space $(\Omega, \mathcal{F})$.

Let

$$
q=\left(q\left(x_{1}, x_{2}, \ldots, x_{m}\right)\right), \quad x_{1}, x_{2}, \ldots, x_{m} \in G
$$

be a distribution on $G^{m}$, and

$$
Q_{n}=\left(q_{n}\left(y \mid x_{1}, x_{2}, \ldots, x_{m}\right)\right), \quad x_{1}, x_{2}, \ldots, x_{m}, y \in G, n \geq 1
$$

be $m$-order transition matrices. For any vertex $t \in L_{n}, n \geq 1$, if

$$
\begin{gathered}
Q\left(X_{t}=y \mid X_{1_{t}}=x_{1}, X_{2_{t}}=x_{2}, \ldots, X_{m_{t}}=x_{m} \text { and } X_{\sigma} \text { for } \sigma \wedge t \leq 1_{t}\right) \\
=Q\left(X_{t}=y \mid X_{1_{t}}=x_{1}, X_{2_{t}}=x_{2}, \ldots, X_{m_{t}}=x_{m}\right) \\
=q_{n}\left(y \mid x_{1}, x_{2}, \ldots, x_{m}\right), \forall x_{1}, x_{2}, \ldots, x_{m}, y \in G
\end{gathered}
$$

and

$$
\begin{aligned}
& Q\left(X_{-(m-1)}=x_{1}, \ldots, X_{-1}=x_{m-1}, X_{o}=x_{m}\right) \\
& =q\left(x_{1}, \ldots, x_{m-1}, x_{m}\right), \quad x_{1}, \ldots, x_{m} \in G
\end{aligned}
$$

then $\left\{X_{t}, t \in T\right\}$ is called a $G$-valued $m$ th-order nonhomogeneous Markov chain indexed by an $m$ rooted Cayley tree with the initial $m$ dimensional distribution (5) and $m$-order transition matrices (6) under the probability measure $Q$, or called a $T$-indexed $m$ th-order nonhomogeneous Markov chain under the probability measure $Q$.

We denote

$$
\begin{aligned}
& o_{m}=\{o,-1,-2, \ldots,-(m-1)\}, o_{m}^{\prime}=\{-1,-2, \ldots,-(m-1)\}, \\
& X_{1}^{n}(t)=\left\{X_{n_{t}}, \ldots, X_{2_{t}}, X_{1_{t}}\right\}, X_{0}^{n}(t)=\left\{X_{n_{t}}, \cdots, X_{2_{t}}, X_{1_{t}}, X_{t}\right\}
\end{aligned}
$$

and denote by $x_{1}^{n}(t)$ and $x_{0}^{n}(t)$ the realizations $X_{1}^{n}(t)$ and $X_{0}^{n}(t)$, respectively.

Let $\left\{X_{t}, t \in T\right\}$ be an $m$ th-order nonhomogeneous Markov chains indexed by an $m$ rooted Cayley tree $T$ under the probability measure $Q$ defined on above. It is easy to see that

$$
Q\left(x^{T^{(n)}}\right)=Q\left(X^{T^{(n)}}=x^{T^{(n)}}\right)=q\left(x_{-(m-1)}, \ldots, x_{0}\right) \prod_{k=1}^{n} \prod_{t \in L_{k}} q_{k}\left(x_{t} \mid x_{1}^{m}(t)\right) .
$$

In the following, we always assume that $P\left(x^{T}\left({ }^{n}\right)\right), Q\left(x^{T}\left({ }^{n}\right)\right), q\left(x_{1}, \ldots, x_{m}\right)$, and $\left\{q_{n}(y \mid\right.$ $\left.\left.x_{1}, \ldots, x_{m}\right), n \geq 1\right\}$ are all positive. 
There have been some works on limit theorems for tree-indexed stochastic process. Benjamini and Peres [3] have given the notion of the tree-indexed Markov chains and studied the recurrence and ray-recurrence for them. Berger and Ye [4] have studied the existence of entropy rate for some stationary random fields on a homogeneous tree. Pemantle [5] proved a mixing property and a weak law of large numbers for a PPG-invariant and ergodic random field on a homogeneous tree. Ye and Berger [6,7], by using Pemantle's result and a combinatorial approach, have studied the ShannonMcMillan theorem with convergence in probability for a PPG-invariant and ergodic random field on a homogeneous tree. Yang and Liu [8] have studied a strong law of large numbers for the frequency of occurrence of states for Markov chains field on a Bethe tree (a particular case of tree-indexed Markov chains field and PPG-invariant random field). Yang [9] has studied the strong law of large numbers for frequency of occurrence of state and Shannon-McMillan theorem for homogeneous Markov chains indexed by a homogeneous tree. Yang and Ye [10] have studied the strong law of large numbers and Shannon-McMillan theorem for nonhomogeneous Markov chains indexed by a homogeneous tree. Huang and Yang [11] have studied the strong law of large numbers and Shannon-McMillan theorem for Markov chains indexed by an infinite tree with uniformly bounded degree. Recently, Shi and Yang [12] have also studied some limit properties of random transition probability for second-order nonhomogeneous Markov chains indexed by a tree. Peng et al. [13] have studied a class of strong deviation theorems for the random fields relative to homogeneous Markov chains indexed by a homogeneous tree. Shi and Yang [2] have studied the strong law of large numbers and Shannon-McMillan for the $m$ th-order nonhomogeneous Markov chains indexed by an $m$ rooted Cayley tree. Yang [14] has also studied a class of small deviation theorems for the sequences of $N$-valued random variables with respect to $m$ thorder nonhomogeneous Markov chains.

In this paper, our main purpose is to extend Yang's [14] result to an $m$ rooted Cayley tree. By introducing the sample divergence rate of any probability measure with respect to $m$ th-order nonhomogeneous Markov measure on an $m$ rooted Cayley tree, we establish a class of strong deviation theorems for the arbitrary random fields indexed by that tree with respect to $m$ th-order nonhomogeneous Markov chains indexed by that tree. As corollaries, we obtain the strong law of large numbers and Shannon-McMillan theorem for $m$ th-order nonhomogeneous Markov chains indexed by that tree.

\section{Main Results}

Before giving the main results, we begin with a lemma.

Lemma 1 Let $T$ be an $m$ rooted Cayley tree, $G=\{0,1, \ldots, b-1\}$ be the finite state space. Let $\left\{X_{t}, t \in T\right\}$ be a collection of $G$-valued random variables defined on the measurable space $(\Omega, \mathcal{F})$. Let $P$ and $Q$ be two probability measures on the measurable space $(\Omega, \mathcal{F})$, and let $\left\{X_{t}, t \in T\right\}$ be an $m$ th-order nonhomogeneous Markov chains indexed by tree $T$ under probability measure $Q$. Let $\left\{g_{n}\left(y_{1}, \ldots, y_{m+1}\right), n \geq 1\right\}$ be a sequence of functions defined on $G^{m+1}$. Let $\mathcal{F}_{n}=\sigma\left(X^{T^{(n)}}\right)(n \geq 1)$. Set

$$
F_{n}(\omega)=\sum_{k=1}^{n} \sum_{t \in L_{k}} g_{k}\left(X_{0}^{m}(t)\right)
$$


and

$$
t_{n}(\lambda, \omega)=\frac{e^{\lambda F_{n}(\omega)}}{\prod_{k=1}^{n} \prod_{t \in L_{k}} E_{Q}\left[e^{\lambda g_{k}\left(X_{0}^{m}(t)\right)} \mid X_{1}^{m}(t)\right]} \cdot \frac{q\left(X_{-(m-1)}, \ldots, X_{o}\right) \prod_{k=1}^{n} \prod_{t \in L_{k}} q_{k}\left(X_{t} \mid X_{1}^{m}(t)\right)}{P\left(x^{T^{(n)}}\right)},
$$

where $E_{Q}$ denote the expectation under probability measure $Q$. Then $\left\{t_{n}(\lambda, \omega), \mathcal{F}_{n}, n \geq 1\right\}$ is a nonnegative martingale under probability measure $P$.

Proof The proof is similar to Lemma 3 of Peng et al. [12], so the proof is omitted.

Theorem 1 Let $T$ be an $m$ rooted Cayley tree, $\left\{X_{t}, t \in T\right\}$ be a collection of random variables taking values in $G=\{0,1, \ldots, b-1\}$ defined on the measurable space $(\Omega, \mathcal{F})$. Let $P$ and $Q$ be two probability measures on the measurable space $(\Omega, \mathcal{F})$, such that $\left\{X_{t}, t \in T\right\}$ is an $m$ th-order nonhomogeneous Markov chain indexed by $T$ under $Q$. Let $h(P \mid Q)$ be defined by $(4),\left\{g_{n}\left(y_{1}, \ldots, y_{m+1}\right), n \geq 1\right\}$ be a sequence of functions defined on $G^{m+1}$. Let $c \geq 0$ be a constant. Set

$$
D(c)=\{\omega: h(P \mid Q) \leq c\}
$$

Assume that there exists $\alpha>0$, such that $\forall i^{m} \in G^{m}$,

$$
b_{\alpha}\left(i^{m}\right)=\limsup _{n \rightarrow \infty} \frac{1}{\left|T^{(n)}\right|} \sum_{k=1}^{n} \sum_{t \in L_{k}} E_{Q}\left[e^{a\left|g_{k}\left(X_{0}^{m}(t)\right)\right|} \mid X_{1}^{m}(t)=i^{m}\right] \leq \tau .
$$

Let

$$
A_{t}=\frac{2 \tau}{e^{2}(t-\alpha)^{2}}
$$

where $o<t<a$. Thus, when $0 \leq c \leq t^{2} A_{t}$, we have

$$
\limsup _{n \rightarrow \infty} \frac{1}{\left|T^{(n)}\right|}\left|\sum_{k=1}^{n} \sum_{t \in L_{k}}\left\{g_{k}\left(X_{0}^{m}(t)\right)-E_{Q}\left[g_{k}\left(X_{0}^{m}(t)\right) \mid X_{1}^{m}(t)\right]\right\}\right| \limsup \leq 2 \sqrt{c A_{t}}, \quad P-\text { a.e., } \omega \in D(c) .
$$

In particular,

$$
\lim _{n \rightarrow \infty} \frac{1}{\left|T^{(n)}\right|} \sum_{k=1}^{n} \sum_{t \in L_{k}}\left\{g_{k}\left(X_{0}^{m}(t)\right)-E_{Q}\left[g_{k}\left(X_{0}^{m}(t)\right) \mid X_{1}^{m}(t)\right]\right\}=0, \quad P-\text { a.e., } \omega \in D(0) .
$$

Proof Let $t_{n}(\lambda, \omega)$ be defined by (11). By Lemma $1,\left\{t_{n}(\lambda, \omega), \mathcal{F}_{n}, n \geq 1\right\}$ is a nonnegative martingale under probability measure $P$. By Doob's martingale convergence theorem, we have

$$
\lim _{n \rightarrow \infty} t_{n}(\lambda, \omega)=t(\lambda, \omega)<\infty, \quad P-\text { a.e. }
$$

Hence,

$$
\limsup _{n \rightarrow \infty} \frac{1}{\left|T^{(n)}\right|} \ln t_{n}(\lambda, \omega) \leq 0, \quad P-\text { a.e.. }
$$

We have by (9), (10), (11) and (17)

$$
\limsup _{n \rightarrow \infty} \frac{1}{\left|T^{(n)}\right|}\left[\sum_{k=1}^{n} \sum_{t \in L_{k}}\left\{\lambda g_{k}\left(X_{0}^{m}(t)\right)-\ln E_{Q}\left[e^{\lambda g_{k}\left(X_{0}^{m}(t)\right)} \mid X_{1}^{m}(t)\right]\right\}-\ln \frac{P\left(X^{T^{(n)}}\right)}{Q\left(X^{T(n)}\right)}\right] \leq 0, P \text {-a.e. }
$$


By (4),(12) and (18)

$$
\limsup _{n \rightarrow \infty} \frac{1}{\left|T^{(n)}\right|} \sum_{k=1}^{n} \sum_{t \in L_{k}}\left\{\lambda g_{k}\left(X_{0}^{m}(t)\right)-\ln E_{Q}\left[e^{\lambda g_{k}\left(X_{0}^{m}(t)\right)} \mid X_{1}^{m}(t)\right]\right\} \leq c, \quad P-a . e ., \omega \in D(c) .
$$

This implies that

$$
\begin{gathered}
\limsup _{n \rightarrow \infty} \frac{\lambda}{\left|T^{(n)}\right|} \sum_{k=1}^{n} \sum_{t \in L_{k}}\left\{g_{k}\left(X_{0}^{m}(t)\right)-E_{Q}\left[g_{k}\left(X_{0}^{m}(t)\right) \mid X_{1}^{m}(t)\right]\right\} \\
\leq \limsup _{n \rightarrow \infty} \frac{1}{\left|T^{(n)}\right|} \sum_{k=1}^{n} \sum_{t \in L_{k}}\left\{\ln E_{Q}\left[e^{\lambda g_{k}\left(X_{0}^{m}(t)\right)} \mid X_{1}^{m}(t)\right]-E_{Q}\left[\lambda g_{k}\left(X_{0}^{m}(t)\right) \mid X_{1}^{m}(t)\right]\right\}+c, \quad P-\text { a.e., } \omega \in D(c)
\end{gathered}
$$

Let $|\lambda|<t$. By inequalities In $x \leq x-1(x>0)$ and $e^{x}-1-x \leq \frac{x^{2}}{2} e^{|x|}$, and noticing that

$$
\max \left\{x^{2} e^{-h x}, x \geq 0\right\}=4 e^{-2} / h^{2}(h>0) .
$$

We have

$$
\begin{aligned}
& \limsup _{n \rightarrow \infty} \frac{1}{\left|T^{(n)}\right|} \sum_{k=1}^{n} \sum_{t \in L_{k}}\left\{\ln E_{Q}\left[e^{\lambda g_{k}\left(X_{0}^{m}(t)\right)} \mid X_{1}^{m}(t)\right]-E_{Q}\left[\lambda g_{k}\left(X_{0}^{m}(t)\right) \mid X_{1}^{m}(t)\right]\right\} \\
& \quad \leq \limsup _{n \rightarrow \infty} \frac{1}{\mid T^{(n) \mid}} \sum_{k=1}^{n} \sum_{t \in L_{k}}\left\{E_{Q}\left[e^{\lambda g_{k}\left(X_{0}^{m}(t)\right)} \mid X_{1}^{m}(t)\right]-1-E_{Q}\left[\lambda g_{k}\left(X_{0}^{m}(t)\right) \mid X_{1}^{m}(t)\right]\right\} \\
& \quad \leq \frac{\lambda^{2}}{2} \limsup _{n \rightarrow \infty} \frac{1}{\left|T^{(n)}\right|} \sum_{k=1}^{n} \sum_{t \in L_{k}} E_{Q}\left[g_{k}^{2}\left(X_{0}^{m}(t)\right) e^{\left.|\lambda|\right|_{g_{k}}\left(X_{0}^{m}(t)\right) \mid} \mid X_{1}^{m}(t)\right] \\
& \quad=\frac{\lambda^{2}}{2} \limsup _{n \rightarrow \infty} \frac{1}{\left|T^{(n)}\right|} \sum_{k=1}^{n} \sum_{t \in L_{k}} E_{Q}\left[e^{\alpha\left|g_{k}\left(X_{0}^{m}(t)\right)\right|} g_{k}{ }^{2}\left(X_{0}^{m}(t)\right) e^{(|\lambda|-\alpha)\left|g_{k}\left(X_{0}^{m}(t)\right)\right|} \mid X_{1}^{m}(t)\right] \\
& \quad \leq \frac{\lambda^{2}}{2} \limsup _{n \rightarrow \infty} \frac{1}{\left|T^{(n)}\right|} \sum_{k=1}^{n} \sum_{t \in L_{k}} E_{Q}\left[e^{\alpha\left|g_{k}\left(X_{0}^{m}(t)\right)\right|} 4 e^{-2} /(|\lambda|-a)^{2} \mid X_{1}^{m}(t)\right] \\
& \leq 2 \lambda^{2} \tau / e^{2}(t-\alpha)^{2} .
\end{aligned}
$$

By (20) and (22), we have

$$
\begin{aligned}
& \limsup _{n \rightarrow \infty} \frac{\lambda}{\left|T^{(n)}\right|} \sum_{k=1}^{n} \sum_{t \in L_{k}}\left\{g_{k}\left(X_{0}^{m}(t)\right)-E_{\mathrm{Q}}\left[g_{k}\left(X_{0}^{m}(t)\right) \mid X_{1}^{m}(t)\right]\right\} \\
& \leq \lambda^{2} A_{t}+c, \quad P-\text { a.e., } \omega \in D(c) .
\end{aligned}
$$

When $0<\lambda<t<\alpha$, we have by (23)

$$
\begin{aligned}
& \limsup _{n \rightarrow \infty} \frac{1}{\left|T^{(n)}\right|} \sum_{k=1}^{n} \sum_{t \in L_{k}}\left\{g_{k}\left(X_{0}^{m}(t)\right)-E_{Q}\left[g_{k}\left(X_{0}^{m}(t)\right) \mid X_{1}^{m}(t)\right]\right\} \\
& \leq \lambda A_{t}+c / \lambda, \quad P-\text { a.e., } \omega \in D(c) .
\end{aligned}
$$

It is easy to see that when $0<c<t^{2} A_{t}$, the function $f(\lambda)=\lambda A_{t}+c / \lambda$ attains, at $\lambda=\sqrt{c / A_{t}}$, its smallest value $f\left(\sqrt{c / A_{t}}\right)=2 \sqrt{c A_{t}}$. Letting $\lambda=\sqrt{c / A_{t}}$ in (24), we have

$$
\limsup _{n \rightarrow \infty} \frac{1}{\left|T^{(n)}\right|} \sum_{k=1}^{n} \sum_{t \in L_{k}}\left\{g_{k}\left(X_{0}^{m}(t)\right)-E_{Q}\left[g_{k}\left(X_{0}^{m}(t)\right) \mid X_{1}^{m}(t)\right]\right\} \leq 2 \sqrt{c A_{t}}, \quad P-\text { a.e., } \omega \in D(c) .
$$


When $c=0$, we have by (24)

$$
\limsup _{n \rightarrow \infty} \frac{1}{\left|T^{(n)}\right|} \sum_{k=1}^{n} \sum_{t \in L_{k}}\left\{g_{k}\left(X_{0}^{m}(t)\right)-E_{Q}\left[g_{k}\left(X_{0}^{m}(t)\right) \mid X_{1}^{m}(t)\right]\right\} \leq \lambda A_{t}, \quad P-\text { a.e., } \omega \in D(0) .
$$

Letting $\lambda \rightarrow 0^{+}$in (26), we obtain

$$
\limsup _{n \rightarrow \infty} \frac{1}{\left|T^{(n)}\right|} \sum_{k=1}^{n} \sum_{t \in L_{k}}\left\{g_{k}\left(X_{0}^{m}(t)\right)-E_{Q}\left[g_{k}\left(X_{0}^{m}(t)\right) \mid X_{1}^{m}(t)\right]\right\} \leq 0, \quad P-\text { a.e., } \omega \in D(0) .
$$

Hence, (25) also holds for $c=0$. When $-\alpha<-t<\lambda<0$, by virtue of (23) it can be shown in a similar way that

$$
\liminf _{n \rightarrow \infty} \frac{1}{\left|T^{(n)}\right|} \sum_{k=1}^{n} \sum_{t \in L_{k}}\left\{g_{k}\left(X_{0}^{m}(t)\right)-E_{Q}\left[g_{k}\left(X_{0}^{m}(t)\right) \mid X_{1}^{m}(t)\right]\right\} \geq-2 \sqrt{c A_{t}}, \quad P-\text { a.e., } \omega \in D(c) .
$$

Equation 15 follows from (25) and (28), Equation 15 implies (16) immediately. This completes the proof of the theorem. $\square$

Theorem 2 Let

$$
H_{t}=2 b / e^{2}(t-1)^{2}, \quad 0<t<1
$$

Let $f_{n}(\omega)$ be defined by (2). Under the conditions of Theorem 1 , when $0 \leq c \leq t^{2} H_{t}$, we have

$$
\begin{aligned}
& \quad \limsup _{n \rightarrow \infty}\left\{f_{n}(\omega)-\frac{1}{\left|T^{(n)}\right|} \sum_{k=1}^{n} \sum_{t \in L_{k}} H\left[q_{k}\left(0 \mid X_{1}^{m}(t)\right), \ldots, q_{k}\left(b-1 \mid X_{1}^{m}(t)\right)\right]\right\} \\
& \leq 2 \sqrt{c H_{t}}, \quad P-\text { a.e., } \omega \in D(c) \\
& \liminf _{n \rightarrow \infty}\left\{f_{n}(\omega)-\frac{1}{\left|T^{(n)}\right|} \sum_{k=1}^{n} \sum_{t \in L_{k}} H\left[q_{k}\left(0 \mid X_{1}^{m}(t)\right), \ldots, q_{k}\left(b-1 \mid X_{1}^{m}(t)\right)\right]\right\} \\
& \geq-2 \sqrt{c H_{t}}-c, \quad P-\text { a.e., } \omega \in D(c)
\end{aligned}
$$

where $H\left(p_{0}, \ldots . p_{b-1}\right)$ denote the entropy of distribution $\left(p_{0}, \ldots, p_{b-1}\right)$, i.e.,

$$
H\left(p_{0}, \ldots, p_{b-1}\right)=-\sum_{i=0}^{b-1} p_{i} \ln p_{i}
$$

Proof In Theorem 1, let $g_{k}\left(y_{1}, \ldots, y_{m+1}\right)=-$ In $q_{k}\left(y_{m+1} \mid y_{1}, \ldots, y_{m}\right)$ and $\alpha=1$, we have

$$
\begin{aligned}
E_{Q} & {\left[e^{g_{k}\left(X_{0}^{m}(t)\right)} \mid X_{1}^{m}(t)=i^{m}\right] } \\
& =\sum_{j \in G} e^{\left|-\ln q_{k}\left(j \mid i^{m}\right)\right|} q_{k}\left(j \mid i^{m}\right) \\
& =\sum_{j \in G} q_{k}\left(j \mid i^{m}\right) / q_{k}\left(j \mid i^{m}\right) \\
& =b .
\end{aligned}
$$


Hence, $\forall i^{m} \in G^{m}$,

$$
b_{1}\left(i^{m}\right)=\limsup _{n \rightarrow \infty} \frac{1}{\left|T^{(n)}\right|} \sum_{k=1}^{n} \sum_{t \in L_{k}} E_{Q}\left[e^{g_{k}\left(X_{0}^{m}(t)\right)} \mid X_{1}^{m}(t)=i^{m}\right] \leq b .
$$

Noticing that

$$
\begin{aligned}
& E_{Q}\left[-\ln q_{k}\left(X_{t} \mid X_{1}^{m}(t)\right) \mid X_{1}^{m}(t)\right] \\
& \quad=-\sum_{j \in G} q_{k}\left(j \mid X_{1}^{m}(t)\right) \ln q_{k}\left(j \mid X_{1}^{m}(t)\right) \\
& \quad=H\left[q_{k}\left(0 \mid X_{1}^{m}(t)\right), \ldots, q_{k}\left(b-1 \mid X_{1}^{m}(t)\right)\right] .
\end{aligned}
$$

When $0 \leq c \leq t^{2} H_{t}$, we have by (34),(29) and (15)

$$
\begin{aligned}
& \limsup _{n \rightarrow \infty}\left\{\frac{1}{\left|T^{(n)}\right|} \sum_{k=1}^{n} \sum_{t \in L_{k}}\left(-\ln q_{k}\left(X_{t} \mid X_{1}^{m}(t)\right)\right)-\frac{1}{\left|T^{(n)}\right|} \sum_{k=1}^{n} \sum_{t \in L_{k}} H\left[q_{k}\left(0 \mid X_{1}^{m}(t)\right), \ldots, q_{k}\left(b-1 \mid X_{1}^{m}(t)\right)\right]\right\} \\
& \leq 2 \sqrt{c H_{t}}, \quad P-\text { a.e., } \omega \in D(c) . \\
& \liminf _{n \rightarrow \infty}\left\{\frac{1}{\left|T^{(n)}\right|} \sum_{k=1}^{n} \sum_{t \in L_{k}}\left(-\ln q_{k}\left(X_{t} \mid X_{1}^{m}(t)\right)\right)-\frac{1}{\left|T^{(n)}\right|} \sum_{k=1}^{n} \sum_{t \in L_{k}} H\left[q_{k}\left(0 \mid X_{1}^{m}(t)\right), \ldots, q_{k}\left(b-1 \mid X_{1}^{m}(t)\right)\right]\right\} \\
& \geq-2 \sqrt{c H_{t}}, \quad P-\text { a.e., } \omega \in D(c) .
\end{aligned}
$$

By (35), (9) and $h(P \mid Q) \geq 0$,

$$
\begin{aligned}
& \limsup _{n \rightarrow \infty}\left\{f_{n}(\omega)-\frac{1}{\left|T^{(n)}\right|} \sum_{k=1}^{n} \sum_{t \in L_{k}} H\left[q_{k}\left(0 \mid X_{1}^{m}(t)\right), \ldots, q_{k}\left(b-1 \mid X_{1}^{m}(t)\right)\right]\right. \\
& \leq \limsup _{n \rightarrow \infty}\left\{-\frac{1}{\left|T^{(n)}\right|} \ln P\left(X^{T^{(n)}}\right)-\frac{1}{\left|T^{(n)}\right|} \sum_{k=1}^{n} \sum_{t \in L_{k}}\left(-\ln q_{k}\left(X_{t} \mid X_{1}^{m}(t)\right)\right\}\right. \\
& \quad+\limsup _{n \rightarrow \infty}\left\{\frac { 1 } { | T ^ { ( n ) } | } \sum _ { k = 1 } ^ { n } \sum _ { t \in L _ { k } } \left(-\ln q_{k}\left(X_{t} \mid X_{1}^{m}(t)\right)\right.\right. \\
& \left.\quad-\frac{1}{\left|T^{(n)}\right|} \sum_{k=1}^{n} \sum_{t \in L_{k}} H\left[q_{k}\left(0 \mid X_{1}^{m}(t)\right), \ldots, q_{k}\left(b-1 \mid X_{1}^{m}(t)\right)\right]\right\} \\
& \leq 2 \sqrt{c H_{t}}, \quad P-\text { a.e., } \omega \in D(c) .
\end{aligned}
$$

By (36), (9) and (12), we have

$$
\begin{aligned}
& \liminf _{n \rightarrow \infty}\left\{f_{n}(\omega)-\frac{1}{\left|T^{(n)}\right|} \sum_{k=1}^{n} \sum_{t \in L_{k}} H\left[q_{k}\left(0 \mid X_{1}^{m}(t)\right), \ldots, q_{k}\left(b-1 \mid X_{1}^{m}(t)\right)\right]\right. \\
& \geq \liminf _{n \rightarrow \infty}\left\{-\frac{1}{\left|T^{(n)}\right|} \ln P\left(X^{T^{(n)}}\right)-\frac{1}{\left|T^{(n)}\right|} \sum_{k=1}^{n} \sum_{t \in L_{k}}\left(-\ln q_{k}\left(X_{t} \mid X_{1}^{m}(t)\right)\right\}\right. \\
& \quad+\liminf _{n \rightarrow \infty}\left\{\frac { 1 } { | T ^ { ( n ) } | } \sum _ { k = 1 } ^ { n } \sum _ { t \in L _ { k } } \left(-\ln q_{k}\left(X_{t} \mid X_{1}^{m}(t)\right)\right.\right. \\
& \left.\quad-\frac{1}{\left|T^{(n) \mid}\right|} \sum_{k=1}^{n} \sum_{t \in L_{k}} H\left[q_{k}\left(0 \mid X_{1}^{m}(t)\right), \ldots, q_{k}\left(b-1 \mid X_{1}^{m}(t)\right)\right]\right\} \\
& \geq-h(P \mid Q)-2 \sqrt{c H_{t}} \\
& \geq-2 \sqrt{c H_{t}}-c, \quad P-\text { a.e., } \omega \in D(c) .
\end{aligned}
$$


This completes the proof of this theorem.

Corollary 1 Under the conditions of Theorem 2, we have

$$
\lim _{n \rightarrow \infty}\left\{f_{n}(\omega)-\frac{1}{\left|T^{(n)}\right|} \sum_{k=1}^{n} \sum_{t \in L_{k}} H\left[q_{k}\left(0 \mid X_{1}^{m}(t)\right), \ldots, q_{k}\left(b-1 \mid X_{1}^{m}(t)\right)\right]\right\}=0, \quad P-a . e ., \omega \in D(0) .
$$

If $P<<Q$, then

$$
\lim _{n \rightarrow \infty}\left\{f_{n}(\omega)-\frac{1}{\left|T^{(n)}\right|} \sum_{k=1}^{n} \sum_{t \in L_{k}} H\left[q_{k}\left(0 \mid X_{1}^{m}(t)\right), \ldots, q_{k}\left(b-1 \mid X_{1}^{m}(t)\right)\right]\right\}=0, \quad P-\text { a.e. }
$$

In particular, if $P=Q$,

$$
\lim _{n \rightarrow \infty}\left\{f_{n}(\omega)-\frac{1}{\left|T^{(n)}\right|} \sum_{k=1}^{n} \sum_{t \in L_{k}} H\left[q_{k}\left(0 \mid X_{1}^{m}(t)\right), \ldots, q_{k}\left(b-1 \mid X_{1}^{m}(t)\right)\right]\right\}=0, \quad Q-\text { a.e. }
$$

Proof Letting $c=0$ in (30) and (31), Equation 39 follows. If $P<<$, then $h(P \mid Q)=$ $0, P$ - a.e.,(cf. see [15],P.121), i.e., $P(D(0))=1$. Hence, Equation 40 follows from (39). In particular, if $P=Q$, then $h(P \mid Q) \equiv 0$. Hence, (41) follows from (40).

Theorem 3 Under the conditions of Theorem 1, if $\left\{g_{n}\left(y_{1}, \ldots . y_{m+1}\right), n \geq 1\right\}$ is uniformly bounded, i.e., there exists $M>0$ such that $\left|g_{n}\left(y_{1}, \ldots, y_{m+1}\right)\right| \leq M$, then when $c \geq 0$, we have

$$
\limsup _{n \rightarrow \infty} \frac{1}{\left|T^{(n)}\right|}\left|\sum_{k=1}^{n} \sum_{t \in L_{k}}\left\{g_{k}\left(X_{0}^{m}(t)\right)-E_{Q}\left[g_{k}\left(X_{0}^{m}(t)\right) \mid X_{1}^{m}(t)\right]\right\}\right| \leq M(c+2 \sqrt{c}), \quad P-a . e ., \omega \in D(c) .
$$

Proof By (20) and (12) and the formula in line 2 of (22), we have

$$
\begin{aligned}
& \limsup _{n \rightarrow \infty} \frac{\lambda}{\left|T^{(n)}\right|} \sum_{k=1}^{n} \sum_{t \in L_{k}}\left\{g_{k}\left(X_{0}^{m}(t)\right)-E_{Q}\left[g_{k}\left(X_{0}^{m}(t)\right) \mid X_{1}^{m}(t)\right]\right\} \\
& \quad \leq \limsup _{n \rightarrow \infty} \frac{1}{\left|T^{(n)}\right|} \sum_{k=1}^{n} \sum_{t \in L_{k}} E_{Q}\left[e^{\lambda g_{k}\left(X_{0}^{m}(t)\right)}-1-\lambda g_{k}\left(X_{0}^{m}(t)\right) \mid X_{1}^{m}(t)\right] \\
& \quad+c \quad P-\text { a.e., } \omega \in D(c) .
\end{aligned}
$$

By the hypothesis of the theorem and the inequality $e^{x}-1-x \leq|x|\left(e^{|x|}-1\right)$, we have

$$
e^{\lambda g_{k}\left(X_{0}^{m}(t)\right)}-1-\lambda g_{k}\left(X_{0}^{m}(t)\right) \leq|\lambda| M\left(e^{|\lambda| M}-1\right) .
$$

By (43) and (44)

$$
\begin{aligned}
& \limsup _{n \rightarrow \infty} \frac{\lambda}{\left|T^{(n)}\right|} \sum_{k=1}^{n} \sum_{t \in L_{k}}\left\{g_{k}\left(X_{0}^{m}(t)\right)-E_{Q}\left[g_{k}\left(X_{0}^{m}(t)\right) \mid X_{1}^{m}(t)\right]\right\} \\
& \quad \leq|\lambda| M\left(e^{|\lambda| \mid M}-1\right)+c, \quad P-\text { a.e., } \omega \in D(c) .
\end{aligned}
$$

When $\lambda>0$, we have by (45)

$$
\begin{aligned}
& \limsup _{n \rightarrow \infty} \frac{1}{\left|T^{(n)}\right|} \sum_{k=1}^{n} \sum_{t \in L_{k}}\left\{g_{k}\left(X_{0}^{m}(t)\right)-E_{Q}\left[g_{k}\left(X_{0}^{m}(t)\right) \mid X_{1}^{m}(t)\right]\right\} \\
& \quad \leq M\left(e^{\lambda M}-1\right)+c / \lambda, \quad P-\text { a.e., } \omega \in D(c) .
\end{aligned}
$$


Taking $\lambda=\frac{1}{M} \log (1+\sqrt{c})$, and using the inequality

$$
\log (1+\sqrt{c}) \geq \frac{\sqrt{c}}{1+\sqrt{c}}
$$

we have when $c>0$

$$
\begin{aligned}
& \limsup _{n \rightarrow \infty} \frac{1}{\left|T^{(n)}\right|} \sum_{k=1}^{n} \sum_{t \in L_{k}}\left\{g_{k}\left(X_{0}^{m}(t)\right)-E_{Q}\left[g_{k}\left(X_{0}^{m}(t)\right) \mid X_{1}^{m}(t)\right]\right\} \\
& \leq M \sqrt{c}+\frac{c M}{\log (1+\sqrt{c})} \\
& \leq M(2 \sqrt{c}+c), \quad P-\text { a.e., } \omega \in D(c)
\end{aligned}
$$

When $\lambda<0$, it follows from (45) that

$$
\begin{aligned}
& \liminf _{n \rightarrow \infty} \frac{1}{\left|T^{(n)}\right|} \sum_{k=1}^{n} \sum_{t \in L_{k}}\left\{g_{k}\left(X_{0}^{m}(t)\right)-E_{Q}\left[g_{k}\left(X_{0}^{m}(t)\right) \mid X_{1}^{m}(t)\right]\right\} \\
& \geq-M\left(e^{\lambda M}-1\right)+c / \lambda \quad P-\text { a.e., } \omega \in D(c) .
\end{aligned}
$$

Taking $\lambda=-\frac{1}{M} \log (1+\sqrt{c})$ in (49), and using (47), we have when $c>0$

$$
\begin{aligned}
& \liminf _{n \rightarrow \infty} \frac{1}{\left|T^{(n)}\right|} \sum_{k=1}^{n} \sum_{t \in L_{k}}\left\{g_{k}\left(X_{0}^{m}(t)\right)-E_{Q}\left[g_{k}\left(X_{0}^{m}(t)\right) \mid X_{1}^{m}(t)\right]\right\} \\
& \geq-M \sqrt{c}-\frac{c M}{\log (1+\sqrt{c})} \\
& \geq-M(2 \sqrt{c}+c), \quad P-\text { a.e., } \omega \in D(c) .
\end{aligned}
$$

In a similar way, it can be shown that (48) and (50) also hold when $c=0$. By (48) and (50), we have (42) holds. This completes the proof of this theorem. $\square$

Corollary 2 Under the conditions of Theorem 1, let $g\left(y_{1}, \ldots, y_{m+1}\right)$ be any function defined on $G^{m+1}$. Let $M=\max g\left(y_{1}, \ldots, y_{m+1}\right)$. Then when $c \geq 0$,

$$
\limsup _{n \rightarrow \infty} \frac{1}{\left|T^{(n)}\right|}\left|\sum_{k=1}^{n} \sum_{t \in L_{k}}\left\{g\left(X_{0}^{m}(t)\right)-E_{Q}\left[g\left(X_{0}^{m}(t)\right) \mid X_{1}^{m}(t)\right]\right\}\right| \leq M(c+2 \sqrt{c}), \quad P-\text { a.e., } \omega \in D(c) .
$$

Proof Letting $g\left(y_{1}, \ldots, y_{m+1}\right)=g_{n}\left(y_{1}, \ldots, y_{m+1}\right), n \geq 1$ in Theorem 3, this corollary follows.

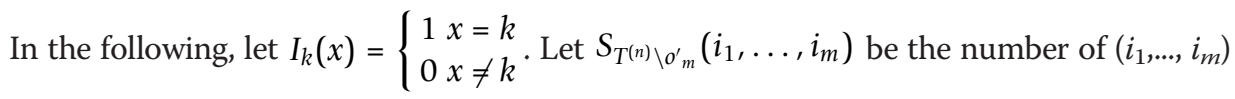
in the collection of $\left\{X_{0}^{m-1}(t), t \in T^{(n)} \backslash o_{m}^{\prime}\right\}$, that is

$$
S_{T^{(n)} \backslash o^{\prime} m}\left(i_{1}, \ldots, i_{m}\right)=\sum_{k=0}^{n} \sum_{t \in L_{k}} I_{i_{1}}\left(X_{(m-1)_{t}}\right) \cdots I_{i_{m}}\left(X_{t}\right),
$$

$S_{T^{(n)} \backslash o_{m}}\left(i_{1}, \ldots, i_{m}, i_{m+1}\right)$ be the number of $\left(i_{1}, \ldots, i_{m}, i_{m+1}\right)$ in the collection of $\left\{X_{0}^{m}(t), t \in T^{(n)} \backslash o_{m}\right\}$, that is 


$$
S_{T^{(n)} \backslash o_{m}}\left(i_{1}, \ldots, i_{m}, i_{m+1}\right)=\sum_{k=1}^{n} \sum_{t \in L_{k}} I_{i_{1}}\left(X_{m_{t}}\right) \cdots I_{i_{m+1}}\left(X_{t}\right) .
$$

Corollary 3 Let $\left\{X_{t}, t \in T\right\}$ be defined as before. Then for all $i_{1}, \ldots, i_{m+1} \in G, c \geq 0$, we have

$$
\begin{aligned}
& \limsup _{n \rightarrow \infty} \mid \frac{S_{T^{(n)} \backslash o^{\prime} m}\left(i_{1}, \ldots, i_{m}\right)}{\left|T^{(n)}\right|}-\frac{1}{\left|T^{(n-1)}\right|} \sum_{l \in G} \sum_{k=0}^{n-1} \sum_{t \in L_{k}} I_{l}\left(X_{(m-1)_{t}}\right) \\
& . I_{i_{1}}\left(X_{(m-2)_{t}}\right) \cdots I_{i_{m-1}}\left(X_{t}\right) q_{k+1}\left(i_{m} \mid l, i_{1}, \ldots, i_{m-1}\right) \mid \leq c+2 \sqrt{c}, \quad P-\text { a.e., } \omega \in D(c) . \\
& \limsup _{n \rightarrow \infty} \mid \frac{S_{T^{(n)} \backslash o_{m}}\left(i_{1}, \ldots, i_{m+1}\right)}{\left|T^{(n)}\right|}-\frac{1}{\left|T^{(n-1)}\right|} \sum_{k=0}^{n-1} \sum_{t \in L_{k}} I_{i_{1}}\left(X_{(m-1)_{t}}\right) \\
& . I_{i_{2}}\left(X_{(m-2)_{t}}\right) \cdots I_{i_{m}}\left(X_{t}\right) q_{k+1}\left(i_{m+1} \mid i_{1}, \ldots, i_{m}\right) \mid \leq c+2 \sqrt{c}, \quad P-\text { a.e., } \omega \in D(c) .
\end{aligned}
$$

Proof Letting $g\left(y_{1}, \ldots, y_{m+1}\right)=I_{i_{1}}\left(y_{2}\right) \cdots I_{i_{m}}\left(y_{m+1}\right)$ in Corollary 2 .

$$
\begin{aligned}
\sum_{k=1}^{n} \sum_{t \in L_{k}} g\left(X_{0}^{m}(t)\right) & =\sum_{k=1}^{n} \sum_{t \in L_{k}} I_{i_{1}}\left(X_{(m-1)_{t}}\right) \cdots I_{i_{m}}\left(X_{t}\right) \\
& =S_{T^{(n)} \backslash o_{m}^{\prime} m}\left(i_{1}, \ldots, i_{m}\right)-I_{i_{1}}\left(X_{-(m-1)}\right) \cdots I_{i_{m}}\left(X_{o}\right),
\end{aligned}
$$

and

$$
\begin{aligned}
& \sum_{k=1}^{n} \sum_{t \in L_{k}} E_{Q}\left[g\left(X_{0}^{m}(t)\right) \mid X_{1}^{m}(t)\right] \\
& \quad=\sum_{k=1}^{n} \sum_{t \in L_{k}} \sum_{x_{t} \in G} g\left(X_{1}^{m}(t), x_{t}\right) q_{k}\left(x_{t} \mid X_{1}^{m}(t)\right) \\
& \quad=\sum_{k=1}^{n} \sum_{t \in L_{k}} \sum_{x_{t} \in G} I_{i_{1}}\left(X_{(m-1)_{t}}\right) \cdots I_{i_{m-1}}\left(X_{1_{t}}\right) I_{i_{m}}\left(x_{t}\right) q_{k}\left(x_{t} \mid X_{1}^{m}(t)\right) \\
& =\sum_{k=1}^{n} \sum_{t \in L_{k}} I_{i_{1}}\left(X_{(m-1)_{t}}\right) \cdots I_{i_{m-1}}\left(X_{1_{t}}\right) q_{k}\left(i_{m} \mid X_{1}^{m}(t)\right) \\
& =\sum_{l \in G} \sum_{k=1}^{n} \sum_{t \in L_{k}} I_{l}\left(X_{m_{t}}\right) I_{i_{1}}\left(X_{(m-1)_{t}}\right) \cdots I_{i_{m-1}}\left(X_{1_{t}}\right) q_{k}\left(i_{m} \mid l, i_{1}, \ldots, i_{m-1}\right) \\
& \quad=N \sum_{l \in G} \sum_{k=0}^{n-1} \sum_{t \in L_{k}} I_{l}\left(X_{(m-1)_{t}}\right) I_{i_{1}}\left(X_{(m-2)_{t}}\right) \cdots I_{i_{m-1}}\left(X_{t}\right) q_{k+1}\left(i_{m} \mid l, i_{1}, \ldots, i_{m-1}\right) .
\end{aligned}
$$

Noticing that $M=\max g\left(y_{1}, \ldots, y_{m+1}\right)=1, \lim _{n \rightarrow \infty} \frac{\left|T^{(n-1)}\right|}{\left|T^{(n)}\right|}=\frac{1}{N}$, by (56) and (57) and Corollary 2, (54) holds. Similarly, we let $g\left(y_{1}, \ldots, y_{m+1}\right)=I_{i_{1}}\left(y_{1}\right) \cdots I_{i_{m+1}}\left(y_{m+1}\right)$, (55) follows.

Corollary 4 Let $\left\{X_{t}, t \in T\right\}$ be defined as before.

$$
\lim _{n \rightarrow \infty} \frac{1}{\left|T^{(n)}\right|} \sum_{k=1}^{n} \sum_{t \in L_{k}}\left\{g\left(X_{0}^{m}(t)\right)-E_{Q}\left[g\left(X_{0}^{m}(t)\right) \mid X_{1}^{m}(t)\right]\right\}=0, \quad P-\text { a.e., } \omega \in D(0),
$$




$$
\begin{aligned}
\lim _{n \rightarrow \infty}\left\{\begin{array}{l}
\frac{S_{T^{(n)} \backslash o_{m}^{\prime}}\left(i_{1}, \ldots, i_{m}\right)}{\left|T^{(n)}\right|}-\frac{1}{\left|T^{(n-1)}\right|} \sum_{l \in G} \sum_{k=0}^{n-1} \sum_{t \in L_{k}} I_{l}\left(X_{(m-1)_{t}}\right) \\
\left.. I_{i_{1}}\left(X_{(m-2)_{t}}\right) \cdots I_{i_{m-1}}\left(X_{t}\right) q_{k+1}\left(i_{m} \mid l, i_{1}, \ldots, i_{m-1}\right)\right\}=0, \quad P-\text { a.e., } \omega \in D(0), \\
\lim _{n \rightarrow \infty}\left\{\frac{S_{T^{(n)} \backslash o_{m}}\left(i_{1}, \ldots, i_{m+1}\right)}{\left|T^{(n)}\right|}-\frac{1}{\left|T^{(n-1)}\right|} \sum_{k=0}^{n-1} \sum_{t \in L_{k}} I_{i_{1}}\left(X_{(m-1)_{t}}\right)\right. \\
\left.. I_{i_{2}}\left(X_{(m-2)_{t}}\right) \cdots I_{i_{m}}\left(X_{t}\right) q_{k+1}\left(i_{m+1} \mid i_{1}, \ldots, i_{m}\right)\right\}=0, \quad P-\text { a.e., } \omega \in D(0) .
\end{array}\right.
\end{aligned}
$$

If $P=Q$, then above equations hold $Q$ - a.e..

Proof Letting $c=0$ in Corollary 2 and Corollary 3, (58)-(60) follow from (51),(54) and (55). In particular, if $P=Q$, then $h(P \mid Q)=0$, so (58)-(60) hold $P$ - a.e., hence hold $Q$ - a.e.

Definition 3 Let $G=\{0,1, \ldots, b-1\}$ be a finite state space and

$$
Q_{1}=\left(q\left(j \mid i^{m}\right)\right), \quad j \in G, i^{m} \in G^{m}
$$

be an $m$ th-order transition matrix. Define a stochastic matrix as follows:

$$
\bar{Q}_{1}=\left(q\left(j^{m} \mid i^{m}\right)\right), \quad i^{m}, j^{m} \in G^{m}
$$

where

$$
q\left(j^{m} \mid i^{m}\right)=\left\{\begin{array}{cc}
q\left(j_{m} \mid i^{m}\right), & \text { if } j_{v}=i_{v+1}, v=1,2, \ldots, m-1, \\
0, & \text { otherwise }
\end{array}\right.
$$

Then $\bar{Q}_{1}$ is called an $m$-dimensional stochastic matrix determined by the $m$ th-order transition matrix. $Q_{1}$.

Lemma 2 (see [16]). Let $\bar{Q}_{1}$ be an $m$-dimensional stochastic matrix determined by the $m$ th-order transition matrix $Q_{1}$. If the elements of $Q_{1}$ are all positive, that is

$$
Q_{1}=\left(q\left(j \mid i^{m}\right)\right), \quad q\left(j \mid i^{m}\right)>0, \forall j \in G, i^{m} \in G^{m}
$$

then $\bar{Q}_{1}$ is ergodic.

Theorem 4 Let $\left\{X_{t}, t \in T\right\}$ be defined as Theorem 1 . Let $S_{T^{(n)} \backslash o^{\prime}{ }_{m}}\left(i_{1}, \ldots, i_{m}\right)=S_{T^{(n)} \backslash o^{\prime} m}\left(i^{m}\right), S_{T^{(n)} \backslash o_{m}}\left(i_{1}, \ldots, i_{m}, i_{m+1}\right)=S_{T^{(n)} \backslash o_{m}}\left(i^{m+1}\right) \quad$ and $\quad f_{n}(\omega)$ defined by (52),(53) and (2), respectively. Let $h(P \mid Q)$ and $D(c)$ be defined by (4) and (12), respectively. Let the $m$ th-order transition matrices defined by (6) be changeless with $n$, that is

$$
Q_{n}=Q_{1}=\left(q\left(j \mid i^{m}\right)\right)
$$

or $\left\{X_{t}, t \in T\right\}$ is an $m$ th-order homogeneous Markov chain indexed by tree $T$ with the $m$ th-order transition matrix $Q_{1}$ under the probability measure $Q$. Let the $m$-dimensional stochastic matrix $\bar{Q}_{1}$ determined by $Q_{1}$ be ergodic. Then for all $i_{1}, \ldots, i_{m+1} \in G$, we have

$$
\lim _{n \rightarrow \infty} \frac{S_{T^{(n)} \backslash o_{m}^{\prime}}\left(i^{m}\right)}{\left|T^{(n)}\right|}=\pi\left(i^{m}\right), \quad P-a . e ., \omega \in D(0) .
$$




$$
\begin{aligned}
& \lim _{n \rightarrow \infty} \frac{S_{T^{(n)} \backslash o_{m}}\left(i^{m+1}\right)}{\left|T^{(n)}\right|}=\pi\left(i^{m}\right) q\left(i_{m+1} \mid i^{m}\right), \quad P-\text { a.e., } \omega \in D(0) . \\
& \lim _{n \rightarrow \infty} f_{n}(\omega)=-\sum_{i^{m} \in G^{m}} \sum_{j \in G} \pi\left(i^{m}\right) q\left(j \mid i^{m}\right) \ln q\left(j \mid i^{m}\right), \quad P-\text { a.e., } \omega \in D(0) .
\end{aligned}
$$

where $\left\{\pi\left(i^{m}\right), i^{m} \in G^{m}\right\}$ is the stationary distribution determined by $\bar{Q}_{1}$.

Proof Proof of Equation 66. Let $k^{m}=\left(k_{1}, \ldots, k_{m}\right)$. If $(65)$ holds, then we have by (63) and (52)

$$
\begin{aligned}
& \sum_{l \in G} \sum_{k=0}^{n-1} \sum_{t \in L_{k}} I_{l}\left(X_{(m-1)_{t}}\right) I_{i_{1}}\left(X_{(m-2)_{t}}\right) \cdots I_{i_{m-1}}\left(X_{t}\right) q_{k+1}\left(i_{m} \mid l, i_{1}, \ldots, i_{m-1}\right) \\
& \quad=\sum_{l \in G} \sum_{k=0}^{n-1} \sum_{t \in L_{k}} I_{l}\left(X_{(m-1)_{t}}\right) I_{i_{1}}\left(X_{(m-2)_{t}}\right) \cdots I_{i_{m-1}}\left(X_{t}\right) q\left(i_{m} \mid l, i_{1}, \ldots, i_{m-1}\right) \\
& =\sum_{l \in G} S_{T^{(n-1)} \backslash o^{\prime} m}\left(l, i_{1}, \cdots, i_{m-1}\right) q\left(i_{m} \mid l, i_{1}, \ldots, i_{m-1}\right) \\
& =\sum_{k^{m} \in G^{m}} S_{T^{(n-1)} \backslash o_{m}^{\prime}}\left(k^{m}\right) q\left(i^{m} \mid k^{m}\right) .
\end{aligned}
$$

By (59) and (69), we have

$$
\lim _{n \rightarrow \infty}\left\{\frac{S_{T^{(n)} \backslash o_{m}^{\prime} m}\left(i^{m}\right)}{\left|T^{(n)}\right|}-\frac{1}{\left|T^{(n-1)}\right|} \sum_{k^{m} \in G^{m}} S_{T(n-1) \backslash o^{\prime} m}\left(k^{m}\right) q\left(i^{m} \mid k^{m}\right)\right\}=0, \quad P-\text { a.e., } \omega \in D(0) .
$$

Multiplying (70) by $q\left(j^{m} \mid i^{m}\right)$, adding them together for $i^{m} \in G^{m}$, and using (70) once again, we have

$$
\begin{aligned}
0 & =\sum_{i^{m} \in G^{m}} q\left(j^{m} \mid i^{m}\right) \cdot \lim _{n \rightarrow \infty}\left\{\frac{S_{T^{(n)} \backslash o_{m}^{\prime}}\left(i^{m}\right)}{\left|T^{(n)}\right|}-\frac{1}{\left|T^{(n-1)}\right|} \sum_{k^{m} \in G^{m}} S_{T^{(n-1)} \backslash o_{m}^{\prime} m}\left(k^{m}\right) q\left(i^{m} \mid k^{m}\right)\right\} \\
& =\lim _{n \rightarrow \infty}\left\{\sum_{i^{m} \in G^{m}} \frac{S_{T^{(n)} \backslash o_{m}^{\prime}}\left(i^{m}\right)}{\left|T^{(n)}\right|} q\left(j^{m} \mid i^{m}\right)-\frac{S_{T^{(n+1)} \backslash o_{m}^{\prime} m}\left(j^{m}\right)}{\left|T^{(n+1)}\right|}\right\} \\
& +\lim _{n \rightarrow \infty}\left\{\frac{S_{T^{(n+1)} \backslash o_{m}^{\prime}}\left(j^{m}\right)}{\left|T^{(n+1)}\right|}-\frac{1}{\left|T^{(n-1)}\right|} \sum_{k^{m} \in G^{m}} S_{T^{(n-1)} \backslash o^{\prime} m}\left(k^{m}\right) \sum_{i^{m} \in G^{m}} q\left(j^{m} \mid i^{m}\right) q\left(i^{m} \mid k^{m}\right)\right\} \\
& =\lim _{n \rightarrow \infty}\left\{\frac{S_{T^{(n+1)} \backslash o_{m}^{\prime}}\left(j^{m}\right)}{\left|T^{(n+1)}\right|}-\frac{1}{\left|T^{(n-1)}\right|} \sum_{k^{m} \in G^{m}} S_{T^{(n-1)} \backslash o^{\prime} m}\left(k^{m}\right) q^{(2)}\left(j^{m} \mid k^{m}\right)\right\}, \quad P-\text { a.e., } \omega \in D(0) .
\end{aligned}
$$

By induction, we have

$$
\lim _{n \rightarrow \infty}\left\{\frac{S_{T^{(n+N)} \backslash o_{m}^{\prime}}\left(j^{m}\right)}{\left|T^{(n+N)}\right|}-\frac{1}{\left|T^{(n-1)}\right|} \sum_{k^{m} \in G^{m}} S_{T^{(n-1)} \backslash o_{m}^{\prime}}\left(k^{m}\right) q^{(N+1)}\left(j^{m} \mid k^{m}\right)\right\}=0, \quad P-\text { a.e., } \omega \in D(0) .
$$

where $q^{(h)}\left(j^{m} \mid k^{m}\right)$ is the $h$ th step probability determined by $\bar{Q}_{1}$. We have by ergodicity

$$
\lim _{N \rightarrow \infty} q^{(N+1)}\left(j^{m} \mid k^{m}\right)=\pi\left(j^{m}\right), \quad \forall k^{m} \in G^{m}
$$

and $\sum_{k^{m} \in G^{m}} S_{T^{(n-1)} \backslash o_{m}^{\prime}}\left(k^{m}\right)=\left|T^{(n-1)}\right|-(m-1)$. (66) follows from (71) and (72). By (66) and (60), Equation 67 follows easily. 
Proof of Equation 68. By (66) and (53), we have

$$
\begin{aligned}
& \left.\sum_{k=1}^{n} \sum_{t \in L_{k}} H\left[q_{k}\left(0 \mid X_{1}^{m}(t)\right), \ldots, q_{k}\left(b-1 \mid X_{1}^{m}(t)\right)\right)\right] \\
& \left.\quad=\sum_{k=1}^{n} \sum_{t \in L_{k}} H\left[q\left(0 \mid X_{1}^{m}(t)\right), \ldots, q\left(b-1 \mid X_{1}^{m}(t)\right)\right)\right] \\
& =-\sum_{k=1}^{n} \sum_{t \in L_{k}} \sum_{j \in G} q\left(j \mid X_{1}^{m}(t)\right) \ln q\left(j \mid X_{1}^{m}(t)\right) \\
& =-\sum_{k=1}^{n} \sum_{t \in L_{k}} \sum_{j \in G} \sum_{i^{m} \in G^{m}} I_{i_{1}}\left(X_{m_{t}}\right) \cdots I_{i_{m}}\left(X_{1_{t}}\right) q\left(j \mid i^{m}\right) \ln q\left(j \mid i^{m}\right) \\
& =-N \sum_{k=0}^{n-1} \sum_{t \in L_{k}} \sum_{j \in G} \sum_{m^{m} \in G^{m}} I_{i_{1}}\left(X_{(m-1)}\right) \cdots I_{i_{m}}\left(X_{t}\right) q\left(j \mid i^{m}\right) \ln q\left(j \mid i^{m}\right) \\
& =-\left.N \sum_{j \in G} \sum_{i^{m} \in G^{m}} S_{T(n-1)}\right|_{o_{m}^{\prime}}\left(i^{m}\right) q\left(j \mid i^{m}\right) \ln q\left(j \mid i^{m}\right) .
\end{aligned}
$$

Noticing that $\lim _{n \rightarrow \infty} \frac{\left|T^{(n-1)}\right|}{\left|T^{(n)}\right|}=\frac{1}{N}$, by (39), (73) and (66), Equation 68 follows. $\square$

\section{Shannon-McMillan Theorem}

Theorem 5 Let $\left\{X_{t}, t \in T\right\}$ be a $G$-valued $m$ th-order nonhomogeneous Markov chain indexed by an $m$ rooted Cayley tree under the probability measure $Q$ with initial distribution (5) and $m$ th-order transition matrices (6). Let $S_{T^{(n)}} \backslash o_{m}^{\prime}\left(i^{m}\right), S_{T^{(n)}} \backslash o_{m}\left(i^{m+1}\right)$ and $f_{n}$ $(\omega)$ be defined as before. Let

$$
Q_{n}=Q_{1}=\left(q\left(j \mid i^{m}\right)\right), \quad q\left(j \mid i^{m}\right)>0, \quad \forall i^{m} \in G^{m}, j \in G,
$$

be another positive $m$ th-order transition matrix. Let $\bar{Q}_{1}$ be an $m$ dimension transition matrix determined by $Q_{1}$. If

$$
\lim _{n \rightarrow \infty} q_{n}\left(j \mid i^{m}\right)=q\left(j \mid i^{m}\right), \quad \forall i^{m} \in G^{m}, j \in G
$$

then

$$
\begin{aligned}
& \lim _{n \rightarrow \infty} \frac{S_{T^{(n)} \backslash o_{m}^{\prime}}\left(i^{m}\right)}{\left|T^{(n)}\right|}=\pi\left(i^{m}\right), \quad P-\text { a.e. } \quad \omega \in D(0), \\
& \lim _{n \rightarrow \infty} \frac{S_{T^{(n)} \backslash o_{m}}\left(i^{m+1}\right)}{\left|T^{(n)}\right|}=\pi\left(i^{m}\right) q\left(i_{m+1} \mid i^{m}\right), \quad P \text { - a.e. } \omega \in D(0), \\
& \lim _{n \rightarrow \infty} f_{n}(\omega)=-\sum_{i^{m} \in G^{m}} \sum_{j \in G} q\left(j \mid i^{m}\right) \ln q\left(j \mid i^{m}\right), \quad P-\text { a.e. } \omega \in D(0),
\end{aligned}
$$

where $\left\{\pi\left(i^{m}\right), i^{m} \in G^{m}\right\}$ is the stationary distribution determined by $\bar{Q}_{1}$. In particular, if $P=Q$, then above equations hold $Q$ - a.e.

Proof By (59), (75), (52) and (66), (76) follows immediately. Similarly, by (60), (75), and (53), (77) follows. It follows from (75) and Cesaro average that

$$
\lim _{n \rightarrow \infty} \frac{1}{\left|T^{(n)}\right|} \sum_{k=1}^{n} \sum_{t \in L_{k}}\left|q_{k}\left(j \mid i^{m}\right) \ln q_{k}\left(j \mid i^{m}\right)-q_{k}\left(j \mid i^{m}\right) \ln q_{k}\left(j \mid i^{m}\right)\right|=0, \quad \forall i^{m} \in G^{m}, j \in G .
$$


Notice that

$$
\begin{aligned}
& \left|\frac{1}{\left|T^{(n)}\right|} \sum_{k=1}^{n} \sum_{t \in L_{k}} H\left[q_{k}\left(0 \mid X_{1}^{m}(t)\right), \ldots, q_{k}\left(0 \mid X_{1}^{m}(t)\right)\right]-\frac{1}{\left|T^{(n)}\right|} \sum_{k=1}^{n} \sum_{t \in L_{k}} H\left[q\left(0 \mid X_{1}^{m}(t)\right), \ldots, q\left(0 \mid X_{1}^{m}(t)\right)\right]\right| \\
& =\mid-\frac{1}{\left|T^{(n)}\right|} \sum_{k=1}^{n} \sum_{t \in L_{k}} \sum_{j \in G} q_{k}\left(j \mid X_{1}^{m}(t)\right) \ln q_{k}\left(j \mid X_{1}^{m}(t)\right)+\frac{1}{\left|T^{(n)}\right|} \sum_{k=1}^{n} \sum_{t \in L_{k}} \sum_{j \in G} q\left(j \mid X_{1}^{m}(t)\right) \ln q\left(j \mid X_{1}^{m}(t)\right) \\
& \leq \frac{1}{\left|T^{(n)}\right|} \sum_{k=1}^{n} \sum_{t \in L_{k}} \sum_{j \in G} \sum_{i^{m} \in G^{m}}\left|q_{k}\left(j \mid i^{m}\right) \ln q_{k}\left(j \mid i^{m}\right)-q\left(j \mid i^{m}\right) \ln q\left(j \mid i^{m}\right)\right| .
\end{aligned}
$$

By (79), (80), (39), (73) and (66), (78) follows. In particular, if $P=Q$, then $h(P \mid Q)=0$. (76), (77) and (78) also holds $P$ - a.e. $\square$

\section{Acknowledgements}

The authors would like to thank the referees for their valuable suggestions and comments. This work is supported by the Research Foundation for Advanced Talents of Jiangsu University (11JDG116) and the National Natural Science Foundation of China (11071104,11171135,71073072).

\section{Author details}

${ }^{1}$ Faculty of Science, Jiangsu University, Zhenjiang 212013, China ${ }^{2}$ Department of Mathematics, Chaohu University, Chaohu 238000, China

\section{Authors' contributions}

ZS, WY and LT carried out the design of the study and performed the analysis. WP participated in its design and coordination. All authors read and approved the final manuscript.

\section{Competing interests}

The authors declare that they have no competing interests.

Received: 21 March 2011 Accepted: 4 January 2012 Published: 4 January 2012

\section{References}

1. Liu, W, Wang, LY: The Markov approximation of the random field on Cayley tree and a class of small deviation theorems. Stat Probab Lett. 63, 113-121 (2003). doi:10.1016/S0167-7152(03)00058-0

2. Shi, ZY, Yang, WG: Some limit properties for the mth-order nonhomogeneous Markov chains indexed by an $\mathrm{m}$ rooted Cayley tree. Stat Probab Lett. 80, 1223-1233 (2010). doi:10.1016/j.spl.2010.03.020

3. Benjamini, I, Peres, Y: Markov chains indexed by trees. Ann Probab. 22, 219-243 (1994). doi:10.1214/aop/1176988857

4. Berger, T, Ye, Z: Entropic aspects of random fields on trees. IEEE Trans Inf Theory. 36, 1006-1018 (1990). doi:10.1109/ 18.57200

5. Pemantle, R: Andomorphism invariant measure on tree. Ann Probab. 20, 1549-1566 (1992). doi:10.1214/aop/1176989706

6. Ye, Z, Berger, T: Ergodic,regularity and asymptotic equipartition property of random fields on trees. Combin Inf Syst Sci. 21, 157-184 (1996)

7. Ye, Z, Berger, T: Information measure for discrete random field. Science, Beijing. (1998)

8. Yang, WG, Liu, W: Strong law of large numbers for Markov chains fields on a bethe tree. Stat Probab Lett. 49, 245-250 (2000). doi:10.1016/S0167-7152(00)00053-5

9. Yang, WG: Some limit properties for Markov chains indexed by a homogeneous tree. Stat Probab Lett. 65, 241-250 (2003). doi:10.1016/j.spl.2003.04.001

10. Yang, WG, Ye, Z: The asymptotic equipartition property for Markov chains indexed by a Homogeneous tree. IEEE Trans Inf Theory. 53(9), 3275-3280 (2007)

11. Huang, $\mathrm{HL}$, Yang, WG: Strong law of large numbers for Markov chains indexed by an infinite tree with uniformly bounded degree. Sci China Ser A Math. 51(2), 195-202 (2008). doi:10.1007/s11425-008-0015-1

12. Shi, ZY, Yang, WG: Some limit properties of random transition probability for second-order nonhomogeneous Markov chains indexed by a tree. J Inequal Appl ID 503203. (2009)

13. Peng, WC, Yang, WG, Wang, B: A class of small deviation theorems for functional of random fields on a homogeneous tree. J Math Anal Appl. 361, 293-301 (2009)

14. Yang, WG: A class of small deviation theorems for the sequences of $N$-valued random variables with respect to $m$ thorder nonhomogeneous Markov chains. Acta Mathematica Scientia 29A(2), 517-527 (2009). in Chinese

15. Gray, RM: Entropy and Information Theory. Springer, New York (1990)

16. Yang, WG, Liu, W: The asymptotic equipartition property for Mth-order nonhomogeneous Markov information sources. IEEE Trans Inf Theory. 50(12), 3326-3330 (2004). doi:10.1109/TIT.2004.838339

doi:10.1186/1029-242X-2012-1

Cite this article as: Shi et al:: A class of small deviation theorems for the random fields on an $m$ rooted Cayley tree. Journal of Inequalities and Applications 2012 2012:1. 